\title{
Underwater multi-target tracking with particle filters
}

\author{
I. Masmitja, S. Gomariz, J. Del \\ Rio \\ SARTI Group. Elect. Department. \\ Universitat Politècnica de Catalunya \\ Vilanova i la Geltrú, Spain. \\ ivan.masmitja@upc.edu
}

\author{
P. J. Bouvet \\ Underwater Acoustics Lab. \\ ISEN Brest YNCREA Ouest, France \\ pierre-jean.bouvet@isen- \\ ouest.yncrea.fr
}

\author{
J. Aguzzi \\ Marine Science Institute (ICM). \\ Consejo Superior de Investigaciones \\ Científica (CSIC). Barcelona, Spain \\ jaguzzi@icm.csic.es
}

\begin{abstract}
Robotic platforms communication and interoperability is of relevance for marine science and industrial monitoring. We present results of a particle filter study based on underwater Multi-Target Tracking (MTT) using Autonomous Underwater Vehicles (AUV). The main goal was to assess the viability of using a single surface vehicle as a mobile landmark to track and follow a fleet of underwater targets, each one equipped with an acoustic tag where the slant ranges between the surface vehicle and the underwater targets are the unique input for the filters.
\end{abstract}

Keywords - tracking; underwater; multi-vehicle; particle filter; collaboration; acoustic communication

Multi-vehicle cooperation and Underwater Sensor Networks (USN) have become one of the main areas of study in marine technology. These new methods, where different sensors, nodes, and vehicles are working together in a collaborative way, open new possibilities to discover and monitor the high complexity of oceanographic phenomena, and to develop new industrial applications. The idea of collaboration among multiple vehicles or standalone sensors to achieve complex tasks, has been a hot topic in the past decade $[1,8]$. Nowadays, this idea is becoming even more and more popular, due to the apparition of cheaper and flexible devices, such as new vehicles and acoustic modems. As an example, technical solutions proposed by the MORPH project [2], include different methods and tools to map the underwater environment using a fleet of autonomous vehicles. The range of opportunities and areas for this technology includes:

- Mining, and oil and gas industries: to create more accurate maps, studies of the seafloor and underwater reservoirs, etc.

- Fisheries: to track spatiotemporal changes in stocks demography and behaviour to preserve quotas, etc.

- Oceanographic research: Eulerian approaches to the study of complex ocean phenomena and seascapes properties in relation to climate change or algal blooms, preserve species or habitats, etc.

However, one of the main drawbacks in multi-target collaboration is the uncertainty in underwater positioning and tracking, which is expensive in terms of power, size, and complexity in both hardware and signal processing. The main reason is due to the underwater medium, which allows poor electromagnetic propagation, and therefore, no GPS or radio frequency systems can be successfully used. Consequently, the acoustic and dead-reckoning methods are primary solutions for underwater positioning and communication. Nevertheless, the traditional Long Base-Line (LBL) method is not valid under dynamic areas of study, as when the target travels thousands of metres. On the other hand, the Ultra Short Base-Line (USBL) devices are, in general, too big to be installed on AUVs or too much expensive. Researches have recently focused on Mobile Long Base-Line (MLBL) methods (also called Virtual Long Base-Line VLBL) to solve those kinds of problems, where different Autonomous Surface Vehicles (ASV) are used as a mobile landmark to estimate the position of underwater devices [3]. However, in general, the MLBL is used as a dead-reckoning aid. The surface vehicles give periodic georeferenced positions to the underwater devices, which have their own positioning system, such as a Doppler Velocity Log (DVL) with an Inertial Measurement Unit (IMU). Nonetheless, this method is not valid in situations where the power consumption, the size and the economic cost of the underwater device are very restrictive. This should be the case of tracking small devices as drifters, small marine species, or to track tens or hundreds of devices, where in this case cost per unit is a key factor.

In this scenario, we have developed an underwater MTT method using range-only and single-beacon strategy based on Particle Filters (PF) algorithm. This paper develops part of the studies presented in [4], but in a multi-target scenario and by introducing more operative aspects as random noise on range measurements, and PF parameters adjustment. Moreover, to make simulation very realistic, the proposed method have been studied using Evologics' modem emulator [6], which allows a close insight on the projected final application with their acoustic modems. These acoustic modems have a physical layer dedicated to measure the transmission and reception times of a data packet with a synchronization pulse, giving these time instants to high level protocols, time synchronized events can be generated by them. Therefore, an accurate Time Of Flight (TOF), and the slant range associated between modems, can be computed $[9,10]$.

\section{METHOD PROPOSED FOR MULTI-TARGET TRACKING}

The main purpose of the study is to follow a set of underwater targets, which can range from acoustic transponders, to AUV fleet or swarms of tagged marine animals. Therefore, we conceived a surface vehicle which is used as the main node in the network (master), and a set of underwater targets which are the secondary nodes (slaves). The master interrogates each slave node periodically to obtain the slant ranges that are used 
as input of the filters to compute the target's localization estimations where each target has its own filter. This scenario is illustrated in Fig. 1.

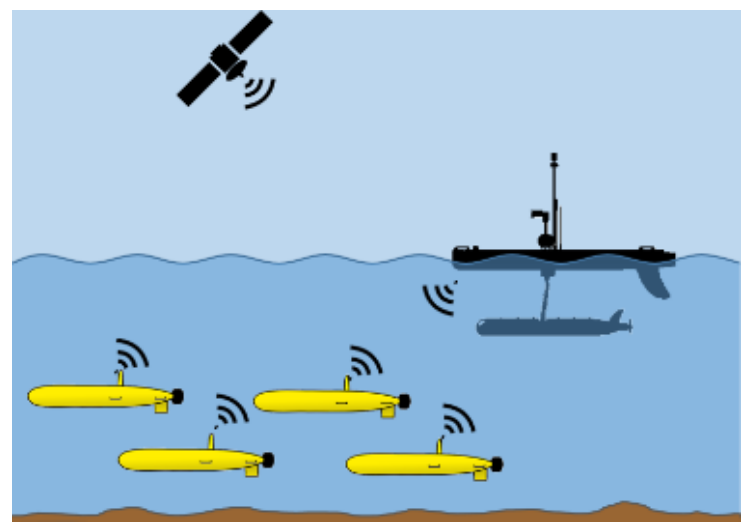

Fig. 1. Range based multi-target tracking scenario.

\section{UNDERWATER TARGET TRACKING}

In [4] we presented a Particle Filter (PF) algorithm for rangeonly and single-beacon target tracking, where an ASV was used to follow a single underwater target. We observe the better performance of the PF in front of the Extended Kalman Filter (EKF). Although the EKF had the best target prediction at the end of the simulation, its setting time was greater than the PF, and therefore, had worst performance.

\section{A. Particle Filter basis}

The main algorithm's steps were described in [4, 7]. However, one of the most important steps in PF is the resampling method, which is essential to obtain a good estimation and avoid the degeneracy problem, but it can increase the computational costs [5]. In this case, we have chosen a compound sampling method to preserve particle diversity. The main group of the particles are resampled using a systematic method (3), whereas some of them are randomly initialized inside a $50 \mathrm{~m}$ radius circumference $(4,5)$, which is centred on the previous target prediction. With this scheme, we want to reduce the degeneracy problem and obtain a better response in front of sudden changes on target forward direction. Finally, the $\mathrm{PF}$ algorithm has to incorporate random noise to increase the particle diversity. In this case, the velocity, orientation, and forward movement noise have been added in the prediction step, which propagate the particles according to motion model. And a sense noise in the measurement step, which updates the particles' weights.

\section{B. Particle Filter applied to multi-target tracking}

The method applied for MTT is based on a bank of filters (Fig. 2), where each filter is used to track a particular target. PF solves, in a non-parametric way, the probability distribution problem of Hidden Markov Models (HMM), given by the recursion of

$$
p\left(x_{t} \mid z_{1: t-1}\right)=\sum_{x_{t-1}} \underbrace{p\left(x_{t} \mid x_{t-1}\right)}_{\text {motion model }} \underbrace{p\left(x_{t-1} \mid z_{1: t-1}\right)}_{\text {particles }},
$$

and

$$
p\left(x_{t} \mid z_{1: t}\right) \propto \underbrace{p\left(z_{t} \mid x_{t}\right)}_{\begin{array}{c}
\text { importance } \\
\text { weights }
\end{array}} \underbrace{p\left(x_{t} \mid z_{1: t-1}\right)}_{\text {particles }},
$$

where $x_{t}$ is the target state at time $t$ and $z_{t}$ is the observation at time $t$. Equation (1) is the motion update, which is composed by a prediction step, where each particle moves according to a motion model, and a measurement step, where then its importance weight is calculated through a measurement of the real world. The second phase (2) is the measurement update, where all particles are resampled according to their importance weights using a combination of systematic and random methods.

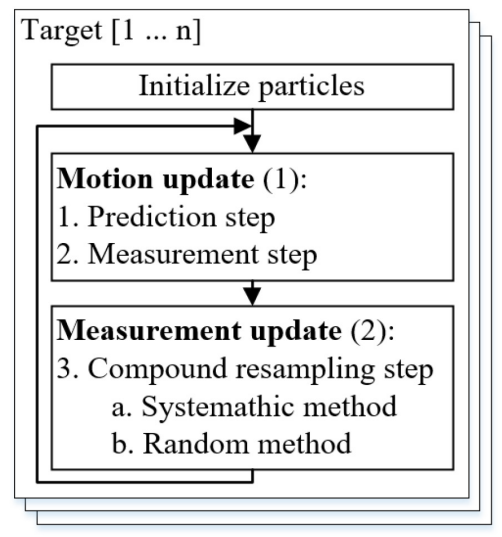

Fig. 2. Block diagram where the bank of PF to compute MTT is represented.

The systematic resampling method is well known, which offers a good performance in terms of computational complexity and resampling quality $[5,11]$. This method uses a vector equal to the cumulative sum of the normalized particle weights, and then a random number $u_{t}^{(1)}$ is drawn from the uniform distribution on $(0,1 /(N-k)]$ interval, and the rest of the $u_{t}$ numbers are obtained deterministically as

$$
\begin{gathered}
u_{t}^{(1)} \sim U\left(0, \frac{1}{N-k}\right], \\
u_{t}^{(n)}=u_{t}^{(1)}+\frac{n-1}{N}, n=2, \ldots, N-k,
\end{gathered}
$$

where $k$ is a constant that define the number of particles which will be resampled using the second method. This vector is used to select a set of particles according to the multinomial distribution.

On the other hand, the random method implemented consist into initialize $k$ particles around the previous target position estimated $\hat{x}_{t-1}$ on a circle shape, where the position of each new particle is defined by

$$
\begin{aligned}
& u_{t}^{(k)}(x)=r \cdot \cos (\alpha)+\hat{x}_{t-1}(x), \\
& u_{t}^{(k)}(y)=r \cdot \sin (\alpha)+\hat{x}_{t-1}(y),
\end{aligned}
$$

where $r \sim U\left(0, r_{\text {max }}\right]$, and $\alpha \sim U(0,2 \pi], r_{\text {max }}$ is the maximum radius defined previously by the user. This strategy is carried out to maintain always some particles nearby the target in all its directions, which improve the PF's time response in front of unexpected target's position variations. 


\section{SimULATIONS AND RESUlTS}

We performed two kinds of simulations: standard simulations through Python, and simulations using the Evologics S2C Underwater Acoustic Modem Emulator [6]. The noise on range's measurement and the target estimation accuracy have been studied using Python simulations, whereas the communication between modems and their medium access protocols have been studied using the Emulator.

\section{A. Python simulation}

On Table I we can observe the EKF and PF performances' under different Gaussian noises added on the range's measurements. We compare the mean and variance error after the filter's stabilization. In this case, 9 targets spread on a grid of $200 \mathrm{~m}$ by $200 \mathrm{~m}$ were used.

TABLE I. FILTERS PERFORMANCE UNDER DIFFERENT NOISE RANGES

\begin{tabular}{|c|c|c|c|c|}
\cline { 2 - 5 } \multicolumn{1}{c|}{} & \multicolumn{4}{c|}{${ }^{2}$ RMSE } \\
\cline { 2 - 5 } \multicolumn{1}{c|}{} & \multicolumn{2}{c|}{ EKF } & Mean & STD \\
\hline Noise STD [m] & Mean & STD & 5.12 & 0.9 \\
\hline 0 & 209.07 & 15.68 & 5.31 & 1.29 \\
\hline 2 & 187.1 & 15.02 & 7.95 & 1.93 \\
\hline 4 & 174.93 & 16.47 & PF \\
\hline
\end{tabular}

a. Simulation using only 9 targets spread on a gird of $200 \mathrm{~m}$ by $200 \mathrm{~m}$, the Root Mean Square Error (RMSE) is the mean of this 9 targets.

These simulations shows that the PF is, much better than the EKF, where both mean and Standard Deviation (STD) of the Root Mean Square Error (RMSE) of all targets' position and their estimations have smaller values. This is an important difference respect to [4], where the EKF was more accurate after the transient response than the PF. However, the initialization point of each filter are worse in this case due to the multi-target scenario, and PF overperforms the EKF in such cases.

The filters' performance are shown in Fig. 3, where the RMSE and its STD for each target is represented, which have been obtained after 10 iterations with a Gaussian noise equal to $2 \mathrm{~m}$. Moreover, Fig. 4 shows one of these iterations on the $x-y$ plane. On these colour maps, the isobars show the error between the real target position and its estimation. In general, for both PF and EKF methods, the error is lower on the middle, where the ASV performs its manoeuvres. However, the PF over performs the EKF method, where its maximum error is less than $5 \mathrm{~m}$, in contrast with the more than $300 \mathrm{~m}$ of error obtained using the EKF.

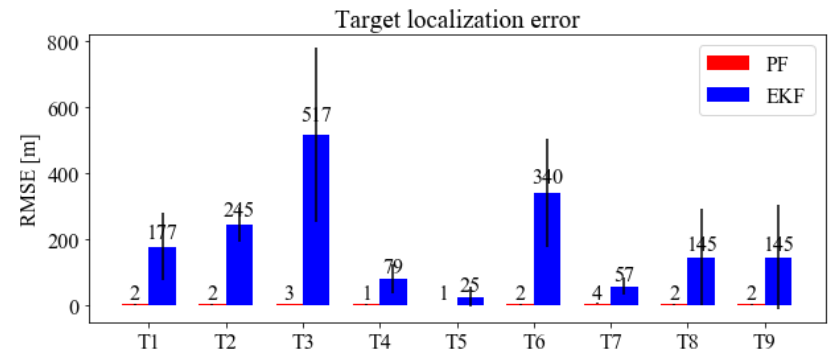

Fig. 3. RMSE and its STD for each target after $25^{\prime}$ of tracking. These values have been obtained after 10 iterations with $2 \mathrm{~m}$ of Gaussian noise added at each range measurement.

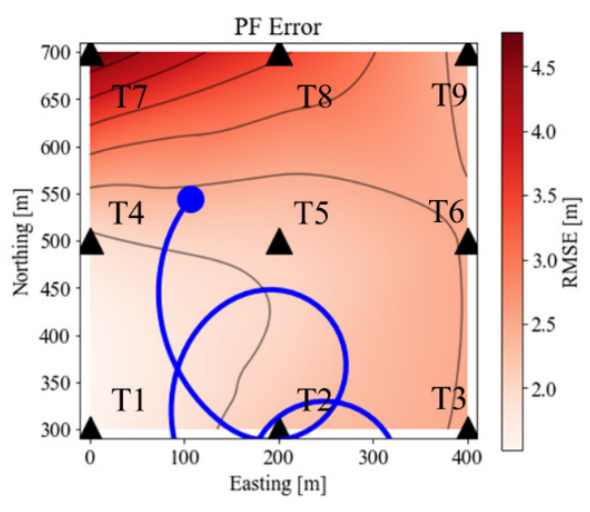

(A)

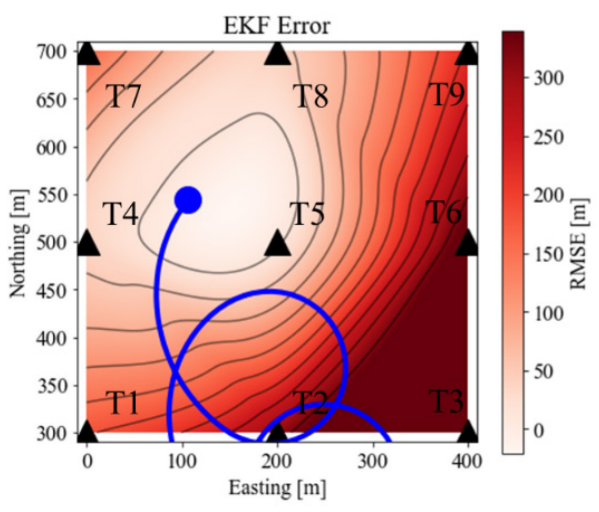

(B)

Fig. 4. RMSE after $1.5 \mathrm{~km}$ of forward movement, with $1 \mathrm{ASV}$ (blue dot) and 9 targets (black triangles). A) using a PF algorithm to estimat each targtet position, and B) using a EKF estimator. The color degradation indicates a target's estimation error that would be obtained if it was in such position. This representation has obtained throught the error of the 9 targets at their position and using a cubic interpolation over the whole grid map.

In both graphs (Fig. 3 and Fig. 4) we can observe that the targets which are on the centre and left-centre are better estimated than the others. This is due the fact that the manoeuvres of the observer are closer to this area. Furthermore, the target number 5 (T5) has the best estimation. In this case, due twofold reasons: all the observer manoeuvres are around this target, which increase its observability; and the filter initialization point was closer to the real target position, because of all initialization points estimate the target position on the centre of the graph, the same position where observer is placed at the beginning.

On the other hand, a set of simulations have been conducted to observe the performance of different resampling algorithms used in the PF estimator. In this case 3 methods have been implemented: Multinomial Resampling (MR), Systematic Resampling (SR), and Compound Resampling (CR). A simulation with 9 targets which have a sudden change in their direction have carried out to appreciate the time response of each method. All the targets were following a south-north straight line, and after 100 iterations their direction changed 90 degrees. The average error of the 9 targets through the time, for each resampling method, is represented in Fig. 5. We can observe that the targets turned at $T_{T}=16.7^{\prime}$. The fastest algorithm to reduce 
the error, and therefore to find the correct target location, was the CR method, which needed $1^{\prime}$ to reduce the error below 30 $\mathrm{m}$. Whereas the MR and SR needed $11.5^{\prime}$ and $9^{\prime}$ respectively.

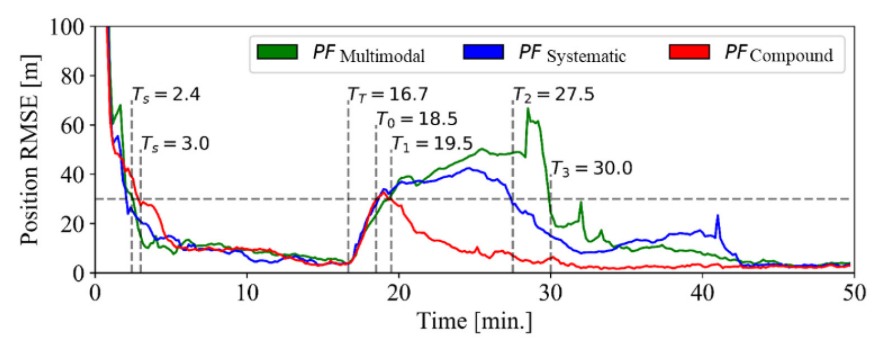

Fig. 5. Avarage of the 9 targes position estimations throuth the time using 3 different PF's resampling strategies. Multimodal, Systematic, and Compound mehtods. Where $T_{T}$ is the turn time instant where all targets changed its direction 90 degrees.

\section{B. Using Evologics modem emulator}

Finally, with the modem emulator tool by Evologics, one can simulate the performance of all the nodes (the master and slaves) working together. This tool allows the user to know the time response of each modem, and simulate a quasi-real scenario in the point of view of the acoustic modems and its communication protocol. We can study the maximum range measurement frequency for each target, and therefore, the scalability of the system. Here we present 2 methods to obtain the range from each target: a first method using simple Query/Answer $(\mathrm{Q} / \mathrm{A})$ strategy; and a second method using Time-Division Multiple Access (TDMA) as a channel access method.

In the $\mathrm{Q} / \mathrm{A}$ strategy, each slant range between the observer and the target is obtained sending an Instant Message (IM) to the target and waiting for its response, as it is represented on Fig. 6. The time delay $T_{d}^{Q / A}$ required to read a range is computed by

$$
\begin{aligned}
T_{d}^{Q / A} & =n T_{a}^{Q / A} \\
T_{d}^{Q / A} & =n 2\left(T O F+T_{T R}+T_{P}\right),
\end{aligned}
$$

where $n$ is the targets' number, TOF is the messages' Time Of Flight, $T_{T R}$ is the transmission time, and $T_{P}$ is the time required to process the IM message.

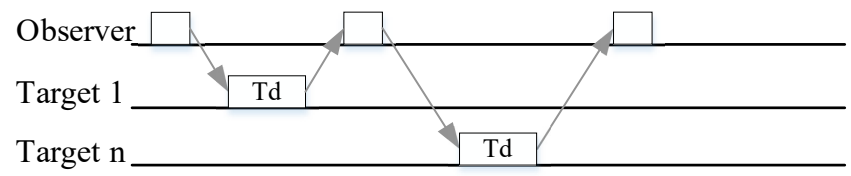

Fig. 6. Query/answer comunication protocol between observer and target modems to obtain their slant range.

On the other hand, using the TDMA method (see Fig. 7) each target sends a response from a broadcast query inside its specific time slot using Synchronous Instant Messages (SIM). The time delay $T_{d}^{T D M A}$ of each target, which is required to allow the transmission inside its slot, is calculated by the linear equation:

$T_{d}^{T D M A}=n T_{a}^{T D M A}+T_{b}^{T D M A}$
$T_{d}^{T D M A}=n\left(2 T O F+T_{T R}+T_{G}\right)+2 T O F+T_{T R}+T_{P}$,

where $T_{G}$ is the guard time between slots. Using these parameters, we make sure that no interferences between transmissions will occur. The TOF has been computed using the maximum distance available between modems.

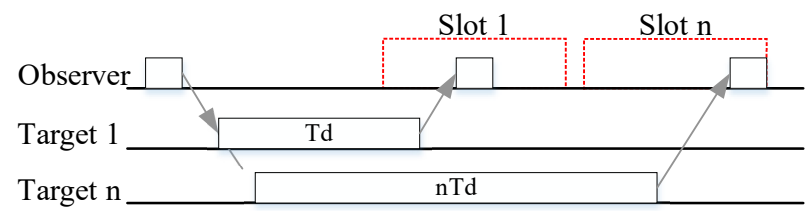

Fig. 7. TDMA comunication protocol between observer and target modems to obtain their slant range.

The results obtained through the modem emulator are presented on Fig. 8, where we can observe the time required to obtain a slant range measurement by both $\mathrm{Q} / \mathrm{A}$ and TDMA methods. Different simulations have been carried out using different number of targets and their maximum distance from the observer.

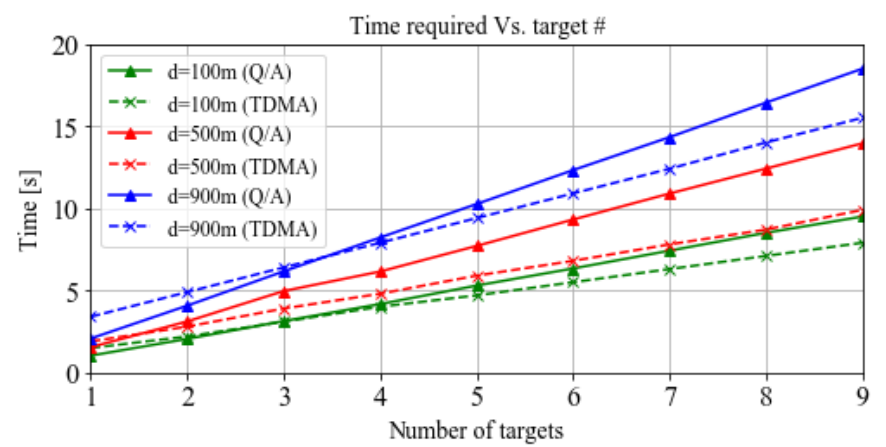

Fig. 8. Time required to obtain a slant range for different number of modems and different ranges between modems and the observer (in this case, at $100 \mathrm{~m}$, $500 \mathrm{~m}$, and $900 \mathrm{~m}$ ). Solid lines represent the $\mathrm{Q} / \mathrm{A}$ method, and discontinuous lines represent the TDMA method.

The linear equation $T_{d}^{Q / A}$ has a greater gradient than the $T_{d}^{T D M A}$ equation. On the other hand, $T_{d}^{T D M A}$ have a non-zero offset. Therefore, we can observe that the $\mathrm{Q} / \mathrm{A}$ method is better than TDMA for a scenario with small number of modems, whereas if the number of modes is bigger (more than 4 ), the TDMA method reduces the Q/A time. For example, the TDMA method can read the range of 9 modems at $500 \mathrm{~m}$ up to $28.6 \%$ faster than $\mathrm{Q} / \mathrm{A}$.

\section{CONCLUSION}

This paper shows the performance of PF as an underwater multi-target tracking method, which is better in noisy and worse initialization scenarios than EKF, as it is shown on Table I and Fig. 3. These simulations demonstrate the good characteristics of the PF to track multiple targets, where an average error equal to $5 \mathrm{~m}$ can be obtained while tracking more than 9 targets at 200 $\mathrm{m}$ of distance between each one.

On the other hand, different simulations have been carried out through Evologics' Modem Emulator, which provides a quasi-real scenario for modem communication. Using this tool, 
we could conduct comparisons between $\mathrm{Q} / \mathrm{A}$ and TDMA methods to read the range from multiple targets, obtaining their performance. We demonstrated that the TDMA method be $28.6 \%$ faster than the $\mathrm{Q} / \mathrm{A}$ method in some scenarios.

To conclude, all the work presented in this paper shows the ability of PF algorithm to track multiple underwater targets from a single observer (i.e. an autonomous surface vehicle).

\section{ACKNOWLEDGMENT}

This work was partially supported by the project JERICONEXT from the European Commission's Horizon 2020 research and Innovation programme under grant agreement No 654410. We also thanks the financial support from the Spanish Ministerio de Economía y Competitividad under contract TEC2017-87861-R project RESBIO. This work has been lead and carried out by members of the Tecnoterra associated unit of the Scientific Research Council through the Universitat Politècnica de Catalunya, the Jaume Almera Earth Sciences Institute and the Marine Science Institute.

\section{REFERENCES}

[1] J. González, I. Masmitjà, S. Gomáriza, E. Molino, J. del Río, A. Mànuel, J. Busquets, A. Guerrero, F. López, M. Carreras, D. Ribas, A. Carrera, C. Candela, P. Ridao, J. Sousa, P. Calado, J. Pinto, A. Sousa, R. Martins, D. Borrajo, A. Olaya, B. Garau, I. González, S. Torres, K. Rajan, M. McCann, J. Gilabert, AUV Based Multi-vehicle Collaboration: Salinity Studies in Mar Menor Coastal Lagoon, In IFAC Proceedings Volumes, Volume 45, Issue 5, 2012, Pages 287-292, ISSN 1474-6670, ISBN 9783902823199 .

[2] Kalwa, Joerg; Tietjen, Daniel; Carreiro-Silva, Marina; Fontes, Jorge; Brignone, Lorenzo; Gracias, Nuno; Ridao, Pere; Pfingsthorn, Max; Birk, Andreas; Glotzbach, Thomas; Eckstein, Sebastian; Caccia, Massimo; Alves, João; Furfa "The European Project MORPH: Distributed UUV
Systems for Multimodal, 3D Underwater Surveys." Marine Technology Society Journal 50 (2016): 26-41.

[3] Chen, Wei, Weisheng Yan, Rongxin Cui and Haiying Cui. "Optimal configuration of USVs for Moving Long Baseline positioning system." 2016 International Conference on Advanced Robotics and Mechatronics (ICARM) (2016): 394-398.

[4] I. Masmitja, P. J. Bouvet, S. Gomariz, J. Aguzzi and J. del Rio, "Underwater mobile target tracking with particle filter using an autonomous vehicle," OCEANS 2017 - Aberdeen, Aberdeen, 2017, pp. $1-5$.

[5] T. Li, M. Bolic and P. M. Djuric, "Resampling Methods for Particle Filtering: Classification, implementation, and strategies," in IEEE Signal Processing Magazine, vol. 32, no. 3, pp. 70-86, May 2015.

[6] Kebkal, O.G.; Kebkal, K.G.; Komar, M. "Development of upper-layer protocols with S2CR acoustic modems emulator." In Proceedings of the Conference on Underwater Communications: Channel Modelling and Validation, UCOMMS, Sestri Levante, Italy, September 2012.

[7] F. Gustafsson, "Particle filter theory and practice with positioning applications," in IEEE Aerospace and Electronic Systems Magazine, vol. 25 , no. 7, pp. 53-82, July 2010 .

[8] D. Mirza and C. Schurgers, "Collaborative Localization for Fleets of Underwater Drifters," OCEANS 2007, Vancouver, BC, 2007, pp. 1-6.

[9] Kebkal K.G., Kebkal O.G., Glushko E. "Propagation Time Estimation between Underwater Acoustic Modems During Data Exchange via Synchronous Instant Messages," in OCEANS 2014 MTS/IEEE Conference and Exhibition, Taiwan, 2014.

[10] Kebkal K.G., Kebkal O.G., Glushko E., Kebkal V.K., Sebastião L., Pascoal A., Gomes J., Ribeiro J., Silva H., Ribeiro M., Indivery G. "Underwater Acoustic Modems with Integrated Atomic Clocks for OneWay Travel-Time Underwater Vehicle Positioning," in Proceedings of the 4th Underwater Acoustics Conference and Exhibition UACE2017, Skiathos, Greece, pp. 315-323, 2017.

[11] Hol, J., Schon, T., and Gustafsson, F. "On Resampling Algorithms for Particle Filters," In Nonlinear Statistical Signal Processing Workshop, 2006 IEEE, pages 79-82, 2006. 